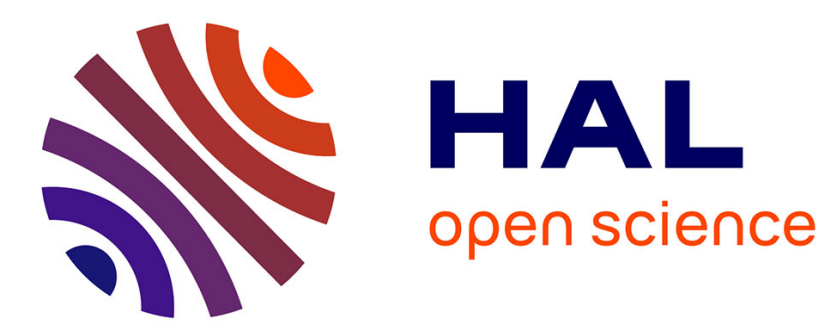

\title{
Automated Refactoring for Size Reduction of CSS Style Sheets
}

Marti Bosch, Pierre Genevès, Nabil Layaïda

\section{To cite this version:}

Marti Bosch, Pierre Genevès, Nabil Layaïda. Automated Refactoring for Size Reduction of CSS Style Sheets. 2014. hal-01021332

\section{HAL Id: hal-01021332 \\ https://hal.inria.fr/hal-01021332}

Preprint submitted on 9 Jul 2014

HAL is a multi-disciplinary open access archive for the deposit and dissemination of scientific research documents, whether they are published or not. The documents may come from teaching and research institutions in France or abroad, or from public or private research centers.
L'archive ouverte pluridisciplinaire $\mathbf{H A L}$, est destinée au dépôt et à la diffusion de documents scientifiques de niveau recherche, publiés ou non, émanant des établissements d'enseignement et de recherche français ou étrangers, des laboratoires publics ou privés. 


\section{Automated Refactoring for Size Reduction of CSS Style Sheets}

\author{
Martí Bosch \\ UPC \& Inria \\ marti.bosch@estudiant.upc.edu
}

\author{
Pierre Genevès \\ CNRS \\ pierre.geneves@cnrs.fr
}

\author{
Nabil Layaïda* \\ Inria \\ nabil.layaida@inria.fr
}

\begin{abstract}
Cascading Style Sheets (CSS) is a standard language for stylizing and formatting web documents. Its role in web user experience becomes increasingly important. However, CSS files tend to be designed from a result-driven point of view, without much attention devoted to the CSS file structure as long as it produces the desired results. Furthermore, the rendering intended in the browser is often checked and debugged with a document instance. Style sheets normally apply to a set of documents, therefore modifications added while focusing on a particular instance might affect other documents of the set.

We present a first prototype of static CSS semantical analyzer and optimizer that is capable of automatically detecting and removing redundant property declarations and rules. We build on earlier work on tree logics to locate redundancies due to the semantics of selectors and properties. Existing purely syntactic CSS optimizers might be used in conjunction with our tool, for performing complementary (and orthogonal) size reduction, toward the common goal of providing smaller and cleaner CSS files.
\end{abstract}

\section{Categories and Subject Descriptors}

1.7 [Document and Text Processing]: Document preparation; D.2 [Software Engineering]: Testing and Debugging

\section{Keywords}

Web development, Style sheets, CSS, Debugging

\section{INTRODUCTION}

Cascading style sheets (CSS) is one of the main components of web development, used to describe the aspect and

Permission to make digital or hard copies of all or part of this work for personal or classroom use is granted without fee provided that copies are not made or distributed for profit or commercial advantage and that copies bear this notice and the full citation on the first page. To copy otherwise, to republish, to post on servers or to redistribute to lists, requires prior specific permission and/or a fee.

Copyright 20XX ACM X-XXXXX-XX-X/XX/XX ...\$15.00.

* Detailed affiliation of authors: M. Bosch ${ }^{1,2,3,4}$, P. Genevès ${ }^{2,1,3}$, N. Layaïda ${ }^{1,2,3}$ with: ${ }^{1}$ Inria; ${ }^{2}$ CNRS, LIG; ${ }^{3}$ Univ. Grenoble Alpes; ${ }^{4}$ Universitat Politècnica de Catalunya. Work partly supported by ANR project Typex (ANR-11-BS02-007). format of a markup document, most of the time an HTML web page. The simplicity of its syntax makes it attractive for designers and amateurs as it only requires assigning values to properties under certain elements of the document.

However CSS language presents a set of combinatorial features that empower its possibilities, while inevitably making it more complex. A single document might use several style sheets, include embedded CSS under the style HTML element, or even use inline styles set directly as attributes of the element concerned. Furthermore, the language used to write selectors is very expressive. The combination of these features might lead to a series of semantical errors and redundancies that make it difficult to spot the origin of problems when one does not get the desired output in the browser. The tool we propose is aimed to help web developers in that matter, as well as reducing the style sheet size.

\section{RELATED WORKS}

In sharp contrast with existing debugging tools [5, 6, 7, 4], we propose a method for automatically analysing and refactoring a CSS file, with the guarantee that the rendering in the browser will not be affected, for any possible document that might use the CSS. A highly novel aspect of our tool is that it is capable of performing CSS refactoring by a semantical analysis of a given CSS file, in the absence of any other information (such as a particular document instance). For this purpose, we build on our previous logical modeling of CSS selectors introduced in [2]. The main difference between the present work and [2] is that [2] focuses on the detection of rendering bugs, whereas the present work seeks to perform automatic CSS size reduction while preserving the rendering semantics.

\section{CSS ANALYSIS AND REFACTORING}

In CSS, rules are encapsulated by a selector, that points to the set of elements that will be affected by the rule's declarations. We borrow ideas from the fields of set theory and tree logics to analyze the sets of elements pointed by the different selectors present in a CSS file. Our tool is concerned with the detection of semantical relations between CSS selectors. When some of these relations are detected, our tool might determine that a property declaration is unnecessary and it will thus be deleted, based on the specificity of selectors. In CSS, a selector's specificity is a vector of four integers $(a, b, c, d)$, where $a=1$ if the property is declared in a style attribute ( $a=0$ otherwise), $b$ is the number of id attributes (of the form "\# ") in the selector, $c$ is the number of other 
attributes and pseudo-classes in the selector, and $d$ is the number of element names and pseudo-elements in the selector. Our tool exploits the facts that: (1) if selectors of two different rules have the same specificity, then the last rule in the style sheet gains precedence; (2) when several selectors point to the same set of elements, then the declarations under the one with higher specificity gain precedence.

\subsection{Containment of selectors}

One fundamental relation between two CSS selectors is containment. For example we say that " $u l>l i$ " is contained into "li" since any "li" element with an "ul" parent is indeed a " $l i$ " element. The existence of containment relations is determined by the analysis of the nested structures of elements and the sets of attributes carried by elements. A selector such as "p. someclass" is contained into "p", since any "p" element with "class" attribute "someclass" is indeed a "p" element. These two kinds of containment can occur simultaneously as it is the case with "table td\#someid" $\subset$ "td". More generally, containment relates sets of pointed elements that are associated with selectors.

Given two selectors $S_{b}$ and $S_{p}, S_{b}$ is contained in $S_{p}$ iff any element pointed by $S_{b}$ is also pointed by $S_{p}$. In this section we treat only proper containment, which means $S_{b} \subset S_{p}$ and not $S_{b} \subseteq S_{p}$. Under these circumstances, for each property declared under both selectors, there are two different procedures according to the selector's specificity:

Refactoring 1. Subset more specific: delete the property declaration from $S_{b}$ only if it has the same value set under both $S_{b}$ and $S_{p}$.

Refactoring 2. Subset less specific: delete the property declaration from $S_{b}$, since the value set under $S_{p}$ will always override the one under $S_{b}$.

For example, consider the following code snippet:

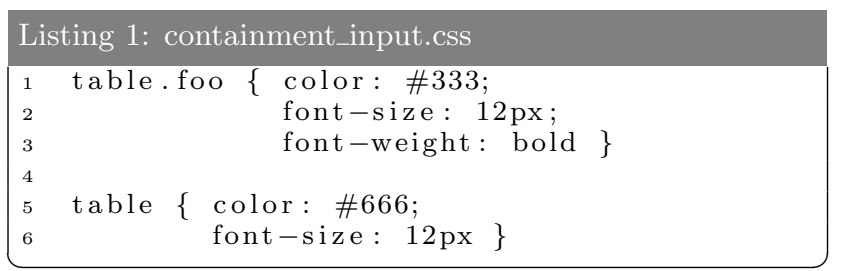

Note that "table.foo" $\subset$ "table" and the subset has a higher specificity $(0,0,1,1)$ against $(0,0,0,1)$ from the superset, so we are in the case of optimization 1. Consequently, we have to preserve the "color: \#333" declaration as it will override the one from "table" when both rules apply. On the other hand, the "font-size" property statement can be removed from the subset as the same value is already pulled from the superset "table". The following code corresponds to the output of the analysis:

\begin{tabular}{|c|}
\hline Listing 2: containment_output.css \\
\hline $\begin{array}{cc}1 & \text { table.foo }\left\{\begin{array}{c}\text { color: } \# 333 ; \\
2\end{array}\right. \\
& \text { font-weight: bold }\} \\
3 & \\
4 & \text { table }\left\{\begin{array}{c}\text { color }: \# 666 ; \\
5\end{array}\right. \\
\text { font-size: } 12 \mathrm{px}\}\end{array}$ \\
\hline
\end{tabular}

If "table" was more specific than "table.foo", optimization 2 would apply and the "color" property declaration could be erased from the subset too as its value would always be overridden by the dominant "\#666" set in the superset.

\subsection{Equivalence of selectors}

Another relation between CSS selectors that can lead to some refactoring is equivalence. Two or more CSS selectors can be equivalent in several ways. Is not uncommon to find a rule $R_{i}$ with a selector such as "body", and later in the same file another rule $R_{j}$ with the same selector "body". Another case of equivalence could be a class selector ".classname" and the attribute selector "[class='classname']", or any similar case with the dual syntax for id attributes. These examples could be studied with basic string processing, but the logical and semantical analysis of selectors allows us to detect more complex equivalences too such as the one between selectors "p:nth-child (odd), p:nth-child (even)" and "p", as every paragraph is either odd or even.

Given two selectors $S_{i}$ and $S_{j}$, they are equivalent iff any element pointed by $S_{i}$ is also pointed by $S_{j}$ and vice-versa. In this context there is only one procedure over the bodies:

Refactoring 3. For each property declared under both selectors, delete the statements under the less specific selector.

To illustrate the analysis, let's look at the next listing:

\begin{tabular}{l} 
Listing 3: equivalence_input.css \\
\begin{tabular}{|rr|r|}
1 & div\#bar \{font-style: italic \\
2 & border: none $\}$ \\
3 & div[id='bar'] & $\{$ color: \#666; \\
4 & font-style: normal \\
5 & border: none $\}$ \\
6 &
\end{tabular} \\
\hline
\end{tabular}

In this case we have two equivalent selectors, "div\#bar" and "div[id='bar']" whose specificities are $(0,1,0,1)$ and $(0,0,1,1)$ respectively. For the properties declared under both rules, the values from "div\#bar" will dominate, so the ones under "div[id='bar']" will never apply, as selectors point to the same set of elements. This means that for "font-style" and "border", the declarations can be safely erased from "div[id='bar']", resulting in the following output:

\begin{tabular}{l} 
Listing 4: equivalence_output.css \\
$\begin{array}{|rr|}1 & \text { div\#bar }\{\text { font-style: italic } \\
2 & \text { border: none }\} \\
3 & \text { div [id='bar'] }\{\text { color: \#666 }\} \\
4 & \text { did }\end{array}$ \\
\hline
\end{tabular}

\subsection{Inheritance of properties}

Whenever containment or equivalence relations are detected between selectors, several selectors point to the same elements, and specificity decides which declarations get precedence. In the context of inheritance, an element might be pointed by only one selector and yet be affected by declarations outside the concerning rule. This is because the declaration has been propagated from some ancestor through the inheritance mechanism. Consider a selector using a descendant combinator " $E_{a n c}>E_{\text {desc }}$ " with a inheritable property 
declaration $P_{a}: V_{a}$, and another selector " $E_{a n c}$ " with the same declaration. This statement might then be redundant as for $E_{\text {desc }}$ the property might inherited from $E_{\text {desc }}$.

However we do not know if some document using this CSS file, presents a structure in which there is a certain element $E_{x}$ placed in between $E_{a n c}$ and $E_{d e s c}$, and the property declarations for $E_{x}$ alter the property inheritance among $E_{\text {anc }}$ and $E_{\text {desc }}$. For example, a selector concerning only an attribute, such as "[input]", is free to be applied to any element on the document. Only certain CSS properties are inherited by default. Therefore, in our analyses, the amount of refactoring due to inheritance is a priori limited.

\section{IMPLEMENTATION TECHNIQUES}

\subsection{Reasoning over selectors}

Given two selectors, we intend to automatically check whether some containment or equivalence relation holds between them. For this purpose, we use the translation of CSS selectors into the tree logic described in [2] and obtain logical formulas. We then formulate containment as logical implication, and test the formula for satisfiability using the logical solver of [3]. For two selectors $S_{1}$ and $S_{2}$, Table 1 summarizes the tests performed, the four possible scenarios obtained according to the results, and the corresponding actions performed, as explained in section 3.1 and 3.2 .

\begin{tabular}{|c|c|c|c|}
\hline $\boldsymbol{S}_{\mathbf{1}} \subseteq \boldsymbol{S}_{\mathbf{2}}$ & $\boldsymbol{S}_{\mathbf{1}} \supseteq \boldsymbol{S}_{\mathbf{2}}$ & Relation & Action \\
\hline 0 & 0 & None & None \\
\hline 0 & 1 & $\boldsymbol{S}_{\mathbf{1}} \supset \boldsymbol{S}_{\mathbf{2}}$ & Refactoring 1, 2 \\
\hline 1 & 0 & $\boldsymbol{S}_{\mathbf{1}} \subset \boldsymbol{S}_{\mathbf{2}}$ & Refactoring 1, 2 \\
\hline 1 & 1 & $\boldsymbol{S}_{\mathbf{1}} \Leftrightarrow \boldsymbol{S}_{\mathbf{2}}$ & Rafactor 3 \\
\hline
\end{tabular}

Table 1: Actions associated with detected relations.

\subsection{Processing on declaration blocks}

Once a relation between selectors $S_{1}$ and $S_{2}$ has been found, for each of the properties declared under both rules, we determine whether it is necessary or not, depending on three aspects: the relation between selectors, the selector's specificity, and whether the properties share the same value; as illustrated on examples in sections 3.1 and 3.2.

In some cases, the deletion of unnecessary property statements results in an empty rule. In this case, the whole rule is (safely) erased from the style sheet.

\subsection{Optimization of elapsed time}

In a style sheet with $n$ rules, each rule can be tested against all rules but itself, adding up to a total of $n \times(n-1)$ possible tests. Given the diversity of elements in a HTML tree, tests concerning selector pairs such as "body" and "p" will not be uncommon. Only by adding a few basic pre checks, we will be able to determine the result of logical tests before actually processing it. We take advantage of two main observations for drastically reducing the number of pairwise tests:

1. If two selectors point to elements with syntactically different names, they will never be contained in each other, in any of the two possible containment directions.
2. If a selector $S_{1}$ refers to one or more attributes that $S_{2}$ does not, $S_{1}$ will never contain $S_{2}$.

\subsection{Statistics tracking}

The tool tracks some statistics about the analysis. First, while parsing it detects the total number of rules, the number of ignored ones, the number of possible tests, and the tests that were actually carried out. After all reasoning is done, the tool counts the number of relations between selectors, the modified rules, the number of deleted properties as well as the deleted bits. Finally, the time spent on each one of the analysis parts is also shown.

\subsection{Room for improvements}

Our prototype implements the aforementioned procedures for a significant CSS subset, sufficient for performing practical experiments with real-world style sheets. Our prototype can be improved in many respects, though. In particular, the library css-validator ${ }^{1}$, that is used for parsing the CSS file and traversing the properties of the rules, could be improved. Some methods concerning comparisons of properties are not implemented, and thus some potential property deletions cannot be automatically carried out. It does not support browser specific properties yet.

Some CSS selectors' features are not supported yet, such as grouping, pseudo-classes, pseudo-elements, multiple class and id selectors and media queries. Consequently, the concerning selectors are ignored. With their implementation, additional refactoring might be performed.

\section{EXPERIMENTAL RESULTS}

In order to get a representative collection of results, three different groups have been defined. The first group involves CSS code provided by frameworks and CMS, and is represented by Bootstrap, Joomla and JQuery. The second group consists in style sheets from complex web applications, and features Instagram, Twitter and The Times. Finally, we have extracted CSS files from some random web sites of medium complexity, which are ACM DL, DocEng, and Inovallée. Table 2 provides detailed information about the corresponding CSS file sizes and complexity.

\begin{tabular}{|c|c|c|}
\hline Name & \# bytes & \# rules \\
\hline Bootstrap (Framework's CSS) & 127343 & 805 \\
Joomla (Template Beez20's CSS) & 30158 & 325 \\
JQuery (Framework's CSS) & 32891 & 349 \\
\hline Instagram (www.instagram.com) & 123815 & 791 \\
The Times (www.thetimes.co.uk) & 89362 & 469 \\
Twitter (www.twitter.com) & 245473 & 2402 \\
\hline ACM DL (dl.acm.org) & 11151 & 97 \\
DocEng (www.doceng2014.org) & 204970 & 1571 \\
Inovallée (www.inovallee.com) & 29930 & 189 \\
\hline
\end{tabular}

Table 2: Dataset for the experiments.

\subsection{CSS Size Reduction in Practice}

\footnotetext{
$\overline{{ }^{1} \text { see http://jigsaw.w3.org/css-validator/ }}$
} 
After processing the aforementioned files, the tool has spotted on average $4.95 \%^{2}$ of unnecessary properties, modifying $4.56 \%$ of the total rules. Of the relations found, $83.38 \%$ were containment ones, and the remaining $16.62 \%$ correspond to equivalence between selectors.

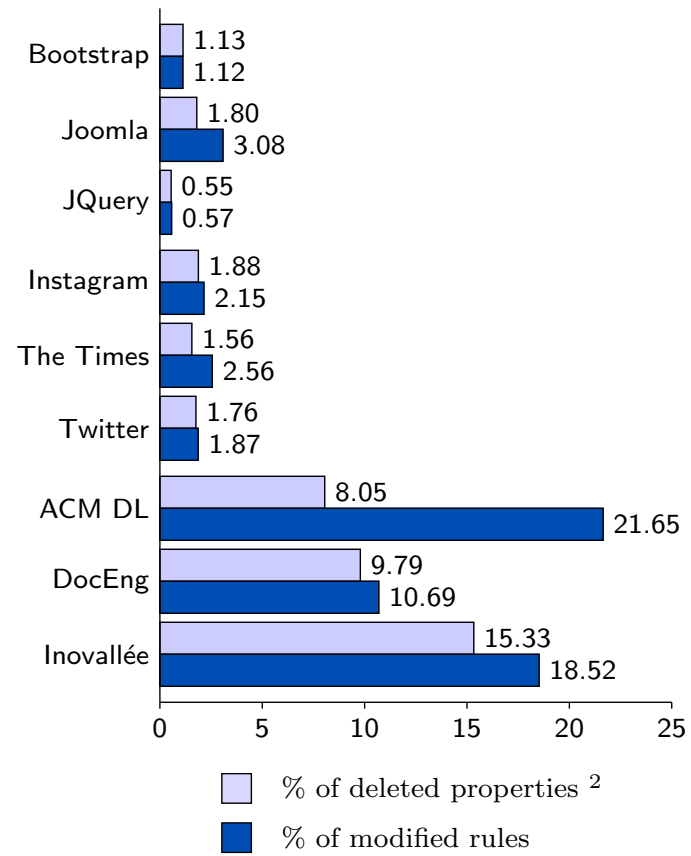

Figure 1: Refactoring performed.

It is clear that the style sheets from the first and second group present a low percentage of deleted properties $(1.16 \%$ and $1.73 \%$ respectively). The same holds for the percentage of modified rules (1.59\% for the first group and $2.19 \%$ for the second one). However, in the third group these numbers rise dramatically, reaching $11.05 \%$ of deleted properties and $16.95 \%$ of modified rules. Although these latter sites might not have involved as much testing as the first ones, they are not amateur web sites either.

\subsection{Performance of the tool}

The time taken to analyze each file is shown in Figure 2. A $34.30 \%$ of the rules have been ignored due to unsupported selectors commented in Section 4.5, and an average of $99.88 \%^{3}$ of the them has been discarded by the optimization mechanism described in Section 4.3. Each test between selectors requires a median of $156.71 \mathrm{~ms}$, so without the optimization mechanism, each file analysis would have taken a median of 16.60 hours, in contraposition to the 78.16 seconds that were actually required, still guaranteeing the same results.

\section{CONCLUSION}

This paper presents a tool that automatically detects and removes unnecessary property declarations in CSS files, based on the analysis of semantical relations between selectors. We provide a first prototype implementation, with many perspectives for further development. Our method can constitute the core mechanism in several applications aimed to help developers code higher quality style sheets. A basic

\footnotetext{
${ }^{2}$ calculated over the properties that the tool supports.
}

${ }^{3}$ calculated over considered tests, discarding ignored ones.

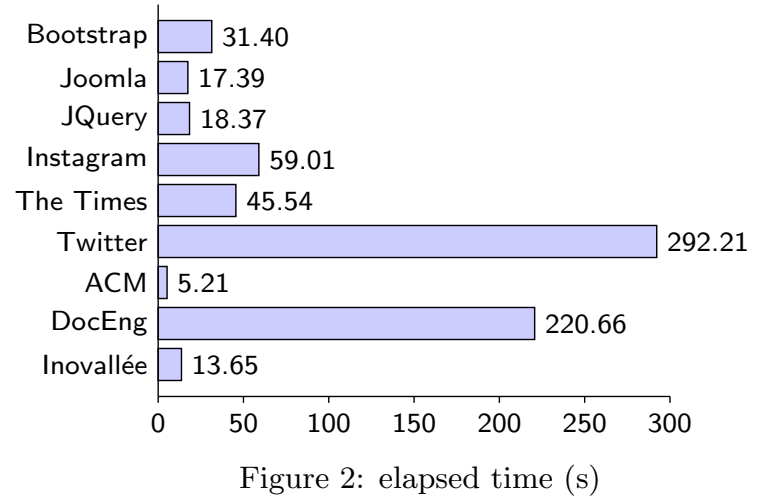

example could be a file processor where the user inputs a CSS file and gets as output an equivalent lighter file. The tool could also be integrated into more powerful components such as context features for web IDEs. In any scenario, the benefit of our tool is to conduct precise semantical analyses, that go far beyond the capabilities of purely syntactic analyses done by current CSS optimizers. Generating equivalent but simpler CSS files not only improves the time spent in loading and formatting a web page, but also facilitates the debugging process of style sheets.

Despite the large number of unsupported features, the results obtained in section 5 already validate our approach: we have been able to detect large numbers of unnecessary property declarations in non-amateur web pages; and we have also found mistakes in the style sheets of some of the most popular web sites. The number of safe modifications can easily grow as more components of CSS are supported and more features are implemented, such as property inheritance, translation of pseudo-classes into query languages, analysis of media queries, merging of equivalent selectors or containment involving grouped selectors.

A perspective for further work consists in extending the number and the precision of our analyses by supporting constraints on the document structure when they are available (as a DTD/Schema, or via another formalism such as [1]).

\section{REFERENCES}

[1] E. Benson and D. R. Karger. Cascading tree sheets and recombinant HTML: better encapsulation and retargeting of web content. In $W W W^{\prime} 13$, pages 107-118, 2013.

[2] P. Genevès, N. Layaïda, and V. Quint. On the analysis of cascading style sheets. In $W W W^{\prime} 12$, pages $809-818$, 2012.

[3] P. Genevès, N. Layaïda, and A. Schmitt. Efficient static analysis of XML paths and types. In PLDI '07, pages 342-351, 2007.

[4] Google. Chrome developer tools, May 2014. https://developer.chrome.com/devtools/.

[5] H.-S. Liang, K.-H. Kuo, P.-W. Lee, Y.-C. Chan, Y.-C. Lin, and M. Y. Chen. SeeSS: Seeing what i broke visualizing change impact of cascading style sheets (CSS). In UIST'13, pages 353-356, 2013.

[6] A. Mesbah and S. Mirshokraie. Automated analysis of CSS rules to support style maintenance. In ICSE'12, pages 408-418, 2012.

[7] Mozilla. Firebug, May 2014. https://getfirebug.com/. 